\title{
Guidance of sentinel lymph node biopsy decisions in patients with T1-T2 melanoma using gene expression profiling
}

\author{
John T Vetto*,1, Eddy C Hsueh², Brian R Gastman³, Larry D Dillon ${ }^{4}$, Federico A Monzon ${ }^{5}$, \\ Robert W Cook ${ }^{5}$, Jennifer Keller ${ }^{2}$, Xin Huang ${ }^{6}$, Andrew Fleming ${ }^{6}$, Preston Hewgley ${ }^{6}$, \\ Pedram Gerami ${ }^{7,8,9}$, Sancy Leachman ${ }^{10}$, Jeffrey D Wayne ${ }^{7,8,11}$, Adam C Berger ${ }^{12}$ \& Martin D \\ Fleming ${ }^{6}$ \\ ${ }^{1}$ Division of Surgical Oncology, Knight Cancer Institute, Oregon Health \& Science University, Portland, OR 97239, USA \\ ${ }^{2}$ Department of Surgery, St Louis University, St Louis, MO 63110, USA \\ ${ }^{3}$ Department of Plastic Surgery, Cleveland Clinic Lerner Research Institute, Cleveland, OH 44915, USA \\ ${ }^{4}$ Larry D Dillon Surgical Oncology \& General Surgery, Colorado Springs, CO 80907, USA \\ ${ }^{5}$ Castle Biosciences, Inc., Friendswood, TX 77546, USA \\ ${ }^{6}$ Division of Surgical Oncology, Department of Surgery, The University of Tennessee Health Science Center, Memphis, TN 38163 , \\ USA \\ ${ }^{7}$ Department of Dermatology, Northwestern University Feinberg School of Medicine, Chicago IL 60611, USA \\ ${ }^{8}$ Skin Cancer Institute, Northwestern University, Lurie Comprehensive Cancer Center, Chicago, IL 60611, USA \\ ${ }^{9}$ Department of Pathology, Northwestern University Feinberg School of Medicine, Chicago IL 60611, USA \\ ${ }^{10}$ Department of Dermatology, Knight Cancer Institute, Oregon Health \& Science University, Portland, OR 97239, USA \\ ${ }^{11}$ Department of Surgical Oncology, Northwestern University Feinberg School of Medicine, Chicago, IL 60611, USA \\ ${ }^{12}$ Department of Surgery, Thomas Jefferson University Hospital, Philadelphia, PA 19017, USA \\ *Author for correspondence: Tel.: 503494 5501; vettoj@ohsu.edu
}

\begin{abstract}
Aim: Can gene expression profiling be used to identify patients with T1-T2 melanoma at low risk for sentinel lymph node (SLN) positivity? Patients \& methods: Bioinformatics modeling determined a population in which a 31-gene expression profile test predicted $<5 \%$ SLN positivity. Multicenter, prospectively-tested $(n=1421)$ and retrospective $(n=690)$ cohorts were used for validation and outcomes, respectively. Results: Patients 55-64 years and $\geq 65$ years with a class $1 \mathrm{~A}$ (low-risk) profile had SLN positivity rates of $4.9 \%$ and $1.6 \%$. Class 2B (high-risk) patients had SLN positivity rates of $30.8 \%$ and $11.9 \%$. Melanoma-specific survival was $99.3 \%$ for patients $\geq 55$ years with class $1 \mathrm{~A}, \mathrm{~T} 1-\mathrm{T} 2$ tumors and $55.0 \%$ for class $2 \mathrm{~B}$, SLN-positive, T1-T2 tumors. Conclusion: The 31-gene expression profile test identifies patients who could potentially avoid SLN biopsy.
\end{abstract}

First draft submitted: 10 December 2018; Accepted for publication: 14 January 2019; Published online: 29 January 2019

Keywords: biomarker $\bullet$ gene expression profiling $\bullet$ melanoma $\bullet$ prognosis $\bullet$ sentinel lymph node biopsy

Cutaneous melanoma (CM) has one of the fastest rising incidence rates of all malignancies in the USA, with more than 91,270 new cases and 9000 deaths expected in 2018 [1]. Of these, more than 70,000 patients are diagnosed with stage I or II (localized) disease as defined by the American Joint Committee on Cancer (AJCC) staging system [1,2]. Patients with early stage melanoma (i.e., AJCC stage I-II) are regarded as having a good prognosis according to population-based risk estimates. However, due to the greater number of individuals within these earlier stages, more than twice as many people diagnosed with early stage disease will ultimately die of melanoma compared with those diagnosed with stage III melanoma [3,4].

Per guidelines, a sentinel lymph node biopsy (SLNB) procedure should be considered for all patients with stage T1b melanoma and above, along with a subset of T1a patients with high-risk features [5,6]. Patients with a positive SLN are at substantially increased risk for distant metastatic disease and death. The Multicenter Selective Lymphadenectomy Trial (MSLT-I) reported that the SLNB procedure provides prognostic information, but does not appear to improve melanoma-specific survival (MSS), although a subset of patients with intermediate thickness melanoma and microscopic nodal involvement showed improved survival at 10 years post-randomization compared 
with patients in the observation arm who experienced nodal recurrence [4]. Recently, the MSLT-II study also showed no survival benefit associated with completion lymphadenectomy in SLN positive patients [7]. For patients able to tolerate adjuvant therapy, identification of SLN positive disease enables use of immunotherapy or targeted therapy in the adjuvant setting [8-11]. Overall, the likelihood of a positive SLN is reported to be $15-20 \%$ in intermediate thickness tumors $[4,12,13]$, but this varies widely depending on tumor and patient features [14-17]. A $<5 \%$ likelihood for a positive SLN is currently recommended by the National Comprehensive Cancer Network guidelines (NCCN v3.2018, July 2018) as a threshold for not performing this procedure, while recommending consideration of SLNB if the risk is $5-10 \%$, and offering SLNB if the risk is above $10 \%$ [6].

The decision to perform SLNB is not without risk. While the rates are low (5-10\%), the SLNB procedure can be associated with complications such as pain, bleeding, allergic reactions to the dye, wound infection, seromas, deep vein thrombosis, nerve damage and edema $[7,18,19]$. Additionally, the procedure requires a dedicated team that includes nuclear medicine physicians, surgeons and pathologists [20-22] and in most cases involves general anesthesia. Therefore, combined with associated surgical and pathology fees, it has been estimated that the cost of an SLNB can be ten-times that of a basic wide local excision alone, and the cost to identify a single positive SLN in the population of patients with thin tumors can approach 1 million dollars [23]. Therefore, strategies that can help reduce surgical risks and complications and potentially reduce healthcare system costs are necessary, especially in populations with low yield from SLNB procedures.

A 31-gene expression profile (31-GEP) that estimates a CM patient's risk for metastatic disease has been previously reported [24-28]. The test uses a radial basis machine algorithm to classify patients into four risk groups with increasing probability of recurrence: low (class 1A), intermediate (class 1B or 2A) or high (class 2B) risk for developing metastasis within 5 years of diagnosis. Prior studies had shown that patients with a class 1 (class $1 \mathrm{~A}$ or 1B) profiles have lower rates of SLN positivity when compared with class 2 (class $2 \mathrm{~A}$ or $2 \mathrm{~B}$ ) patients, albeit with a positive rate higher than the $5 \%$ threshold currently used to determine SLNB eligibility [26,29]. To determine if the 31-GEP test could identify a population with $<5 \%$ likelihood of a positive SLN, we performed modeling in a large retrospective cohort. The current study reports validation of the use of the 31-GEP test to identify patients with low probability of a positive SLN in a defined patient population from a large prospective cohort.

\section{Methods}

Evaluation of SLN prediction by GEP test

Rules-based and regression analyses to determine significant predictors of SLN positivity and identification of optimal cut-offs to define a population in which gene expression could better predict SLNB results were performed. This evaluation was done using the normalized gene expression data and clinicopathologic variables for 946 archived tumor samples collected as part of the institutional review board (IRB)-approved protocol for initial validation of the 31-GEP test. Evaluation to determine if the current algorithm in the 31-GEP could be improved for the purposes of SLN positivity risk prediction was also performed. We employed predictive modeling with multiple machine learning approaches, including neural networks, self-organizing maps, support vector machine and treebased models, using gene expression alone and gene expression with clinicopathologic features. Additional details regarding the evaluation of alternative algorithms for SLN guidance and results are described in the Supplementary Methods, Results and Supplementary Figure 1.

\section{Patient cohorts}

Following determination of the optimal cut-offs for selecting a population in which the test could identify patients with a low probability of a positive SLN, validation of this approach was performed in two prospectively collected cohorts from 26 dermatologic or tertiary care centers. Cohort 1 consisted of 584 patients enrolled in one of two prospective, ongoing registry studies and a prospective clinical utility study [30,31], all of which had IRB-approved study protocols. Participating centers in these registry studies include dermatology practices and tertiary care centers. Cohort 2 included 837 cases from surgical tertiary care centers who tested patients between 2014 and 2017, for which clinical data and SLNB status were collected under IRB-approved research protocols established at each center. Analyses were performed for all patients in these cohorts as well as for the subset that were T1b and above (eligible) or T1a patients who had the procedure performed (assessed). In order to represent the intended use population and to provide better estimates of SLN positivity rates for each genomic class and age group, results from the two pooled cohorts are also shown. To determine the clinical outcome impact of using this approach to determine whether to do an SLNB or not, long-term clinical outcome data were assessed from 690 patients who 


\begin{tabular}{|c|c|c|c|}
\hline Clinical feature & Cohort $1(n=584)$ & Cohort $2(n=837)$ & p-value \\
\hline \multicolumn{4}{|l|}{ Age (years): } \\
\hline - Median (range) & $61(18-100)$ & $63(12-101)$ & 0.05 \\
\hline \multicolumn{4}{|l|}{ Breslow depth $(\mathrm{mm})$ : } \\
\hline - Median (range) & $1.2(0-18)$ & $1.16(0-60)$ & 0.21 \\
\hline Ulceration present & $19 \%(103 / 554)^{\dagger}$ & $25 \%(204 / 819)^{\ddagger}$ & 0.006 \\
\hline Mitotic rate $\geq 1 / \mathrm{mm}^{2}$ & $59 \%(342 / 584)$ & $64 \%(534 / 836)$ & 0.043 \\
\hline Node status positive & $14 \%(59 / 417)^{\S}$ & $12 \%(85 / 701) \mathbb{I}$ & 0.33 \\
\hline \multicolumn{4}{|l|}{ T Stage: } \\
\hline$-\mathrm{T} 1$ & $44 \%(258 / 584)$ & $42 \%(355 / 837)$ & 0.67 \\
\hline$-\mathrm{T} 2$ & $31 \%(183 / 584)$ & $32 \%(269 / 837)$ & \\
\hline$-\mathrm{T3}$ & $17 \%(101 / 584)$ & $17 \%(139 / 837)$ & \\
\hline$-\mathrm{T} 4$ & $7 \%(42 / 584)$ & $9 \%(74 / 837)$ & \\
\hline GEP class 2 & $24 \%(143 / 584)$ & $29 \%(245 / 837)$ & 0.046 \\
\hline \multicolumn{4}{|c|}{$\begin{array}{l}\text { Cohort } 1 \text { is comprised of patients enrolled in two prospective registry studies from dermatology and surgical practices; cohort } 2 \text { is comprised of prospective } \\
\text { tested patients at surgical tertiary care centers. p-values for dichotomous variables represent Pearson's } \chi^{2} \text { tests; } p \text {-values for the continuous variables } 0 \\
\text { age and Breslow depth represent Wilcoxon } F \text { tests. } \\
\dagger 30 \text { cases did not report whether ulceration was present or absent. } \\
\ddagger 18 \text { cases did not report whether ulceration was present or absent. } \\
\S 167 \text { cases did not undergo sentinel lymph node biopsy. } \\
\mathbb{I} 136 \text { cases did not undergo sentinel lymph node biopsy. } \\
\text { GEP: Gene expression profile. }\end{array}$} \\
\hline
\end{tabular}

had more than 5 years of follow-up or a documented recurrence of melanoma [24,25,27,32]. These patients represent a subset of the 946 patient retrospective cohort described above.

\section{GEP testing}

The 31-GEP test (DecisionDx-Melanoma, Castle Biosciences, Inc., TX, USA) was performed on all of the primary melanoma cases in this study, and the result was correlated with tumor, clinical and nodal outcomes, as noted above. All GEP testing was performed in the Castle Biosciences Inc. CAP-accredited and CLIA-certified laboratory. The development and validation of the 31-GEP test have been previously described [24-30].

\section{Statistical analysis}

All statistical analysis was performed using the statistical analysis package $\mathrm{R}$, version 3.3.2. A two-sided $\mathrm{p}$-value $<0.05$ was considered statistically significant. Pearson's $\chi^{2}$ and Wilcoxon F tests were used for comparing demographic and clinical categorical variables between patient groups. Fisher's exact tests were used for SLN positivity comparisons. Survival curves were estimated using the Kaplan-Meier method and compared by log-rank tests. End points for survival analysis included MSS, overall (OS), distant metastasis-free (DMFS) and recurrence-free (RFS) survival. MSS outcomes required confirmed mortality resulting from melanoma, OS included patients who died from melanoma or other/unknown causes, DMFS included any recurrence beyond the regional nodal basin and RFS included any recurrence from melanoma excluding SLN positivity. For RFS and DMFS, a patient was considered censored if the patient was either lost to follow-up or had experienced a death event at the indicated time.

\section{Results}

Validation cohort demographics

Clinical demographics for validation cohort 1 and cohort 2 indicate these patient groups reflect the general population of melanoma patients (Table 1) [12,13], with median ages of 61 and 63 years, respectively, and SLN positive rates of 14 and $12 \%$, respectively. While ulceration rates, mitosis and GEP class were statistically different between cohorts 1 and $2(\mathrm{p}=0.006, \mathrm{p}=0.043$ and $\mathrm{p}=0.046$, respectively), both cohorts are similar in the distribution of $\mathrm{T}$ stages, node positivity and Breslow thickness, reflecting the target population for use of the GEP test for guiding SLNB. 
Table 2. Sentinel lymph node positivity rates in the independent and combined validation cohorts for all T1/T2 patients

\section{in the study.}

\begin{tabular}{|c|c|c|c|c|c|c|}
\hline Patient age group & $\mathrm{T} 1 / \mathrm{T} 2$ & $\begin{array}{l}\text { SLN positivity (class } \\
\text { 1A) }\end{array}$ & $95 \% \mathrm{Cl}$ (class $1 \mathrm{~A})$ & $\begin{array}{l}\text { SLN positivity (class } \\
\text { 2B) }\end{array}$ & 95\% Cl (class 2B) & p-value \\
\hline \multicolumn{7}{|l|}{ Cohort 1} \\
\hline All & 441 & $4.2 \%(14 / 330)$ & $2.3-7.0 \%$ & $7.4 \%(2 / 27)$ & $1.0-24 \%$ & 0.35 \\
\hline$<55$ & 158 & $6.8 \%(8 / 118)$ & $3.0-12.9 \%$ & $0.0 \%(0 / 5)$ & $0-52 \%$ & $>0.99$ \\
\hline $55-64$ & 111 & $5.7 \%(5 / 88)$ & $1.9-12.8 \%$ & $20 \%(1 / 5)$ & $1.0-72 \%$ & 0.29 \\
\hline$\geq 65$ & 172 & $0.8 \%(1 / 124)$ & $0-4.4 \%$ & $5.9 \%(1 / 17)$ & $0-29 \%$ & 0.23 \\
\hline \multicolumn{7}{|l|}{ Cohort 2} \\
\hline All & 624 & $4.8 \%(22 / 460)$ & $3-7.2 \%$ & $24.5 \%(13 / 53)$ & $13.8-38.3 \%$ & $<0.001$ \\
\hline$<55$ & 212 & $8.2 \%(14 / 171)$ & $4.5-13.4 \%$ & $30 \%(6 / 20)$ & $11.9-54.3 \%$ & 0.009 \\
\hline $55-64$ & 136 & $4.3 \%(4 / 94)$ & $1.2-10.5 \%$ & $37.5 \%(3 / 8)$ & $8.5-75.5 \%$ & 0.01 \\
\hline$\geq 65$ & 276 & $2.1 \%(4 / 195)$ & $0-5.2 \%$ & $16 \%(4 / 25)$ & $4.5-36.1 \%$ & 0.007 \\
\hline \multicolumn{7}{|c|}{ Combined cohorts 1 and 2} \\
\hline All & 1065 & $4.6 \%(36 / 790)$ & $3.2-6.3 \%$ & $18.8 \%(15 / 80)$ & $10.9-29 \%$ & $<0.001$ \\
\hline$<55$ & 370 & $7.6 \%(22 / 289)$ & $4.8-11.3 \%$ & $24.0 \%(6 / 25)$ & $9.4-45.1 \%$ & 0.02 \\
\hline $55-64$ & 247 & $4.9 \%(9 / 182)$ & $2.3-9.2 \%$ & $30.8 \%(4 / 13)$ & $9.1-61.4 \%$ & 0.006 \\
\hline$\geq 65$ & 448 & $1.6 \%(5 / 319)$ & $0.5-3.6 \%$ & $11.9 \%(5 / 42)$ & $4.0-25.6 \%$ & 0.003 \\
\hline
\end{tabular}

\section{SLN probability in GEP subclasses}

The aim of this study was to evaluate whether the GEP test could identify patients with low risk for SLN positivity in the T1-T2 melanoma population who currently would be considered for SLNB based on guidelines. Understanding that a patient has a very low risk for a positive SLN could inform decisions on the SLNB procedure. These include patients with T1a melanomas, who may be considered for SLNB based on adverse features, including uncertain microstaging, lymphovascular invasion, elevated mitotic rate and others [6]. Of note, the majority of the patients with T1a melanoma in this study were managed at surgical centers and were thus being considered for SLNB; $34 \%$ of the T1a cases in this study underwent SLNB. We also evaluated performance in a narrower population that included only patients who had the SLNB procedure (assessed) or were T1b and above (eligible). Of the 1421 total patients included in the validation study, 79\% had a SLNB performed. Overall, 1065 had T1 or T2 thickness (i.e., $\leq 2 \mathrm{~mm}$ thick) tumors. As shown in Table 2, the SLN positivity rate for all patients with T1-T2, class 1A tumors was $4.6 \%$ (4.2\% for cohort 1 and $4.8 \%$ for cohort 2$)$, while the SLN positivity rate for patients with $\mathrm{T} 1-\mathrm{T} 2$, class $2 \mathrm{~B}$ tumors was $18.8 \%$ (7.4\% for cohort 1 and $24.5 \%$ for cohort $2 ; \mathrm{p}<0.001$ for class $1 \mathrm{~A}$ vs class $2 \mathrm{~B}$ in the combined cohort). Patients with class 1B-2A showed intermediate SLN positivity rates (Figure 1A) .

Based on a Loess model fit to the risk of SLN positivity with age, the ages of 55 and 65 were identified as inflection points indicating differential risk. As such, patients in the combined cohort were binned into three groups for analysis: $<55,55-64$ and $\geq 65$ years old. Patients with GEP class 1A, T1-T2 tumors in age groups $<55,55-64$ or $\geq 65$ years had SLN positivity rates of $7.6,4.9$ and 1.6\%, respectively, compared with $24.0,30.8$ and $11.9 \%$ for class $2 \mathrm{~B}$ ( $\mathrm{p}<0.02$ for class $1 \mathrm{~A}$ vs class $2 \mathrm{~B}$ in each age group, Table $2 \&$ Figure $1 \mathrm{~A}$ ). As a group, patients $\geq 55$ years with $\mathrm{T} 1-\mathrm{T} 2$ melanoma and a class $1 \mathrm{~A}$ result had a SLN positivity rate of $2.8 \%$ while those with a class $2 \mathrm{~B}$ result had $16.4 \%$ SLN positivity. Similar results were obtained when the analysis was performed only with patients who were eligible for a SLNB and/or had the procedure performed $(n=838$; Table 3, Figure 1B, Supplementary Table 1).

Survival rates associated with SLN guidance by gene expression profiling

Next, we estimated the survival outcomes of patients $\geq 55$ years old with class $1 \mathrm{~A}, \mathrm{~T} 1-\mathrm{T} 2$ tumors, the target population that would not undergo SLNB. Long-term clinical outcomes from patients previously reported in three clinical validity studies for the 31-GEP test $[24,25,27]$ were used for survival analyses. Long-term outcome (survival rate) comparisons of the 690-patient cohort are comparable to that observed with the recently compiled AJCC 8th edition database (MSS for AJCC 8th edition stage I $=98 \%$, stage II $=90 \%$ and stage III $=77 \%$, compared with MSS for stage I $=99 \%$, stage II $=91 \%$ and stage III $=76 \%$ for the 690 -sample) $[2,32]$. As shown in Figure $2 \mathrm{~A}$, 
(A)

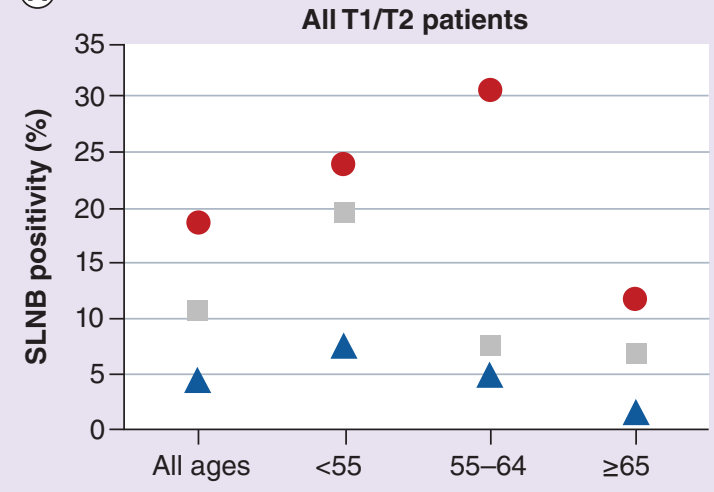

$\Delta$ Class 1A Class 1B/2A Class $2 \mathrm{~B}$

(B)

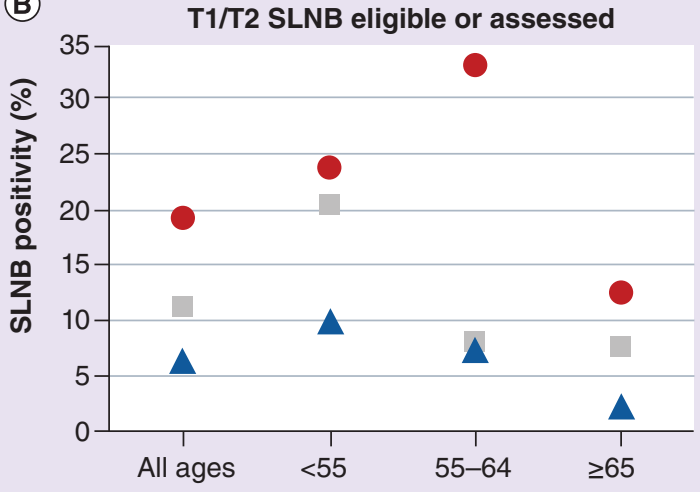

$\Delta$ Class $1 \mathrm{~A} \backsim$ Class $1 \mathrm{~B} / 2 \mathrm{~A} \bullet$ Class $2 \mathrm{~B}$

Figure 1. Sentinel lymph node positivity rate according to gene expression profile, subclass for T1/T2 staged patients of all ages, and stratified in age groups $<55,55-64$ or $\geq 65$ years. (A) Clinical sentinel lymph node positivity rate for T1 or T2 cases within the validation cohort $(n=1065)$, patients $<55$ years old $(n=370)$, between 55 and 64 $(n=247)$ and $\geq 65(n=448)$. These data are also represented in Table 2. (B) Clinical sentinel lymph node positivity rate for T1 or T2 patients who were eligible for or assessed by SLNB. All ages within the validation cohort $(n=838)$, patients $<55$ years old $(n=302)$, between 55 and $64(n=189)$ and $\geq 65(n=347)$ are shown for this eligible or assessed population. These data are also presented in Table 3. In both panels, positivity rates are reported for each GEP test result: class $1 \mathrm{~A}$ (blue triangle), class $1 \mathrm{~B} / 2 \mathrm{~A}$ (gray square), class $2 \mathrm{~B}$ (red circle) results.

SNLB: Sentinel lymph node biopsy.

5-year MSS, OS, DMFS and RFS rates were high for GEP class 1A, but were lower for SLN negative GEP class $2 \mathrm{~B}$ patients, and for SLN positive GEP class $2 \mathrm{~B}$ patients ( $\mathrm{p}<0.05$ for class $1 \mathrm{~A}$ vs class $2 \mathrm{~B}$ and SLN negative for all end points, $\mathrm{p}<0.001$ for class $1 \mathrm{~A}$ vs class $2 \mathrm{~B}$ and SLN positive for all end points). Comparable results were observed when patients of all ages were evaluated (Figure 2B).

\section{Potential impact of gene expression profiling on SLNB rates}

If patients with T1-T2, class $1 \mathrm{~A}$ tumors with a risk of SLN positivity below 5\% would not undergo SLNB, an increase in the yield of positive SLNs and a reduction of the number of SLNB procedures performed would be observed. In this prospective cohort of 1421 patients, if patients 55-64 years with class 1A, T1-T2 tumors did not undergo SLNB, the positive SLN yield in patients in this age group with T1-T4 melanoma would increase from 13.3 to $18.6 \%$, while reducing SLNB procedures by $46.5 \%$ (by $66.7 \%$ in the T1-T2 population alone). The positive SLN yield in patients $\geq 65$ years with T1-T4 melanoma would increase from 8.7 to $13.6 \%$, with a reduction in SLNBs by $42.8 \%$ (by $65.1 \%$ in the T1-T2 population alone). 
Table 3. Sentinel lymph node positivity rates in the independent and combined cohorts for all patients in the study who were eligible and/or assessed with sentinel lymph node biopsy.

\begin{tabular}{|c|c|c|c|c|c|c|}
\hline Patient age group & $\mathrm{T} 1 / \mathrm{T} 2$ & $\begin{array}{l}\text { SLN positivity (class } \\
\text { 1A) }\end{array}$ & $95 \% \mathrm{Cl}$ (class 1A) & $\begin{array}{l}\text { SLN positivity (class } \\
\text { 2B) }\end{array}$ & $95 \% \mathrm{Cl}$ (class 2B) & $p$-value \\
\hline \multicolumn{7}{|l|}{ Cohort 1} \\
\hline All & 326 & $6.2 \%(14 / 226)$ & $3.4-10.2 \%$ & $8.3 \%(2 / 24)$ & $1.0-27 \%$ & 0.66 \\
\hline$<55$ & 128 & $8.9 \%(8 / 90)$ & $3.9-16.8 \%$ & $0 \%(0 / 5)$ & $0-52 \%$ & $>0.99$ \\
\hline $55-64$ & 78 & $8.8 \%(5 / 57)$ & $2.9-19.3 \%$ & $25 \%(1 / 4)$ & $1.0-81 \%$ & 0.35 \\
\hline$\geq 65$ & 120 & $1.3 \%(1 / 79)$ & $0-6.9 \%$ & $6.7 \%(1 / 15)$ & $0-32 \%$ & 0.30 \\
\hline \multicolumn{7}{|l|}{ Cohort 2} \\
\hline All & 512 & $6.3 \%(22 / 349)$ & $4-9.4 \%$ & $24.5 \%(13 / 53)$ & $13.8-38.3 \%$ & $<0.001$ \\
\hline$<55$ & 174 & $10.5 \%(14 / 133)$ & $5.9-17 \%$ & $30 \%(6 / 20)$ & $11.9-54.3 \%$ & 0.027 \\
\hline $55-64$ & 111 & $5.8 \%(4 / 69)$ & $1.6-14.2 \%$ & $37.5 \%(3 / 8)$ & $8.5-75.5 \%$ & 0.022 \\
\hline$\geq 65$ & 227 & $2.7 \%(4 / 147)$ & $0.7-6.8 \%$ & $16 \%(4 / 25)$ & $4.5-36.1 \%$ & 0.017 \\
\hline \multicolumn{7}{|c|}{ Combined cohorts 1 and 2} \\
\hline All & 838 & $6.3 \%(36 / 575)$ & $4.4-8.6 \%$ & $19.5 \%(15 / 77)$ & $11.3-30.1 \%$ & $<0.001$ \\
\hline$<55$ & 302 & $9.9 \%(22 / 223)$ & $6.3-14.6 \%$ & $24 \%(6 / 25)$ & $9.4-45.1 \%$ & 0.046 \\
\hline $55-64$ & 189 & $7.1 \%(9 / 126)$ & $3.3-13.1 \%$ & $33.3 \%(4 / 12)$ & $9.9-65.1 \%$ & 0.016 \\
\hline$\geq 65$ & 347 & $2.2 \%(5 / 226)$ & $0.7-5.1 \%$ & $12.5 \%(5 / 40)$ & $4.2-26.8 \%$ & 0.009 \\
\hline
\end{tabular}

\section{Discussion}

There is a clinical need to identify patients who are likely to have a negative SLNB result in order to reduce surgical burden and cost. Most guidelines suggest considering SLNB for melanomas with pathologic stage T1b and above, as well as T1a melanomas with high-risk features. However, this threshold is not absolute, and physicians weigh many factors, including patient preference, to make decisions pursing SLNB, thus not all eligible patients undergo the procedure [33,34]. Additional information on risk of SLN positivity and clinical outcomes could help in SLNB decision-making.

The association of a 31-GEP class 1 signature with lower SLN positive rates was previously reported by Huang et al. and observed in other cohorts $[26,27,29]$. This suggested that this test could provide information relevant to the likelihood of a tumor to metastasize to the SLN, in addition to the previously demonstrated association of this signature with melanoma recurrence, distant metastasis and death [24-28]. We used modeling in a retrospective cohort of melanoma patients to identify cut-offs for clinicopathologic features that could help identify a patient population in which the test could achieve the goal of identifying patients with $<5 \%$ SLN positivity. This analysis showed that in patients with Breslow thickness $\leq 2.0 \mathrm{~mm}$ (T1-T2) and $\geq 55$ years of age, the current clinical 31-GEP test could achieve this goal. To validate this approach, two contemporary, multicenter study cohorts totaling 1421 prospectively and consecutively tested patients, representative of the general population of melanoma patients who are considered for SLNB [4,15,35], were used. It is important to note that these cohorts include T1a patients, as a subset of this group is currently eligible and contribute to a substantial number of T1-T2 patients who undergo the procedure [6]. Analysis of SEER data (2017 release with AJCC 8th edition staging applied) show that $6.7 \%$ of T1 a patients do undergo a SLNB procedure and since T1a constitutes the largest group of melanoma patients (55.5\% in SEER data), these represent $22.2 \%$ of SLNBs in T1-T2 patients [3]. Similar numbers have been reported by Stiegel et al., who showed that at the Cleveland Clinic, T1a tumors accounted for $21.3 \%$ of all SLNB procedures and $30 \%$ of SLNBs in T1-T2 patients [36].

We investigated whether the 31-GEP test could help identify a patient population that has a $<5 \%$ SLN positive rate and, based on current guideline thresholds, inform decisions on whether to perform the SLNB procedure. The results showed that patients $\geq 55$ years who had a class $1 \mathrm{~A}$ GEP in a T1-T2 tumor had a $2.8 \%$ SLN positive rate, below the $5 \%$ cut-off for recommending the procedure [6]. SEER data indicate that patients who are $\geq 55$ years with T1-T2 melanoma account for approximately $49 \%$ of those who are eligible for SLNB [3], therefore the results of this study have implications for a large number of patients and could help reduce overutilization of healthcare resources for those unlikely to have a positive SLNB. Patients in the Medicare population ( $\geq 65)$ with a class $1 \mathrm{~A}$, 


\section{(A)}

Clinical T1/T2 melanoma (age $\geq 55$ )

5-year survival rates

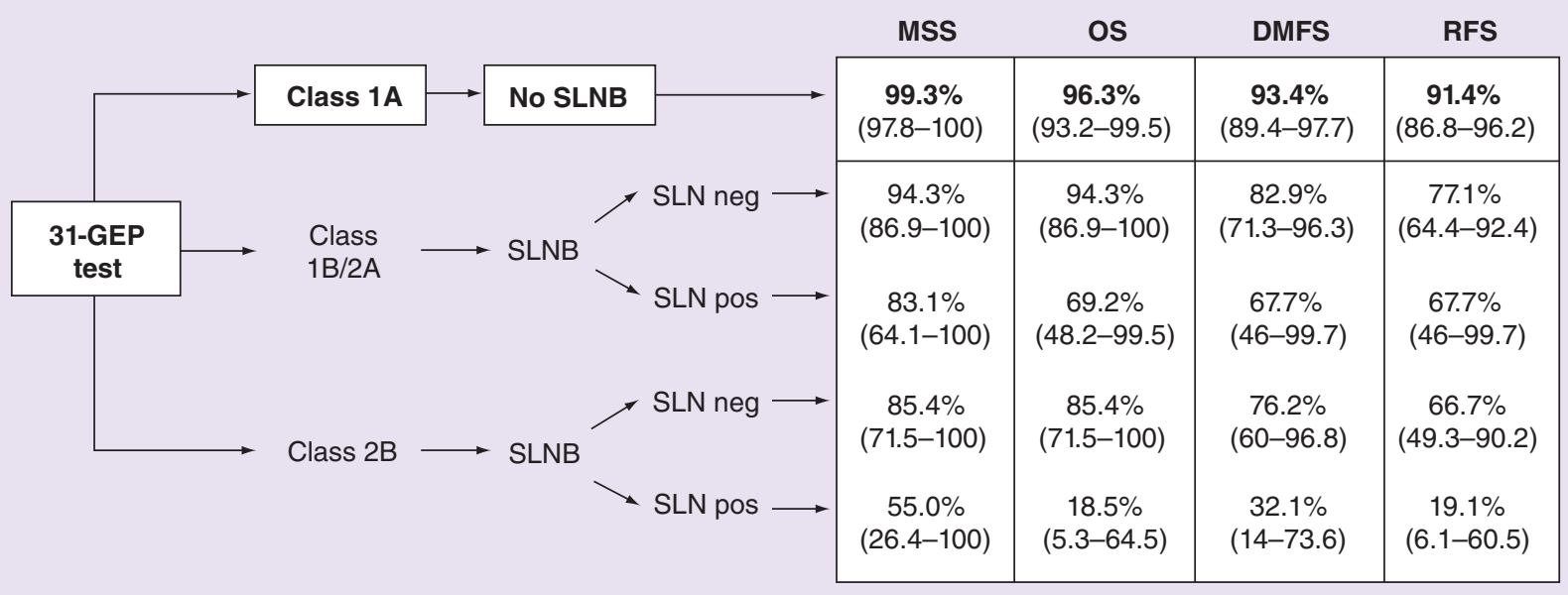

(B)

Clinical T1/T2 melanoma (all ages)

5-year survival rates

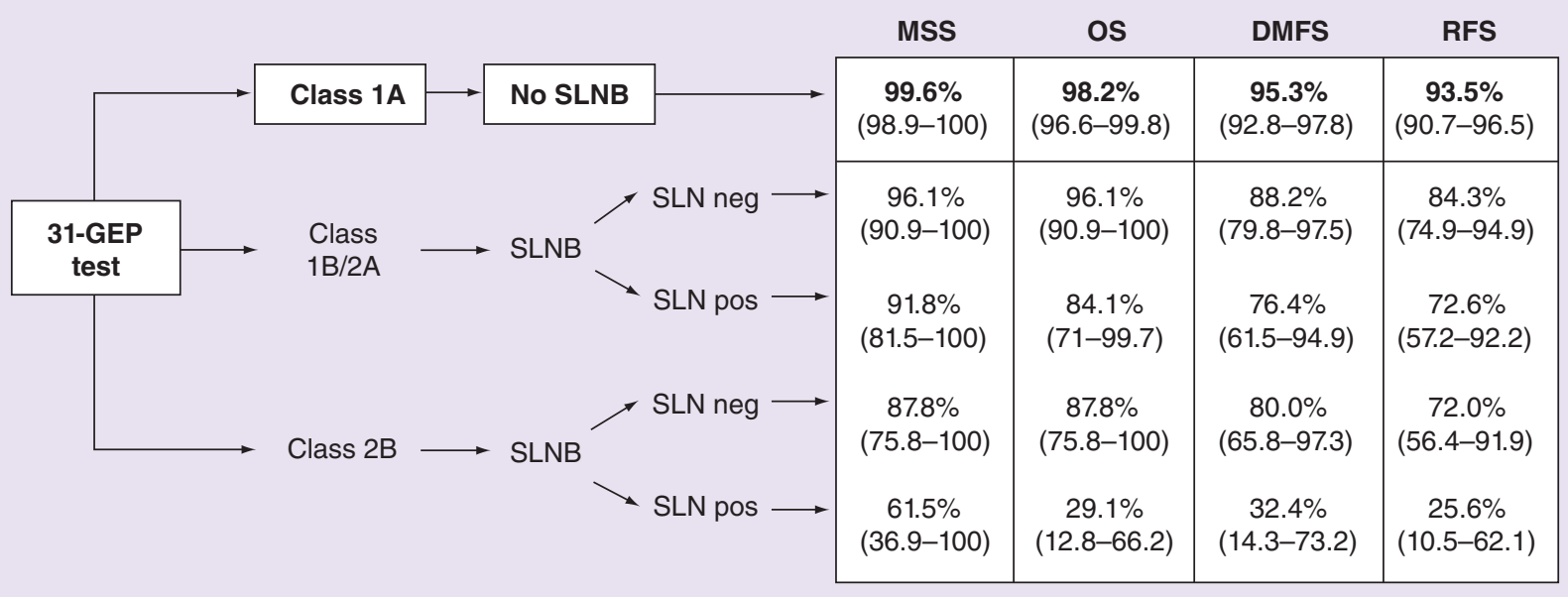

Figure 2. Survival rates according to gene expression profile subclass and sentinel lymph node status for the subset of cases $\geq 55$ years old with T1 or T2 primary tumor thickness in a retrospective patient cohort. (A) Flow chart depicting SLN prediction with the gene expression profile test and outcomes for patients $\geq 55$ years old with a T1 or T2 tumor $(n=224)$ within a retrospective patient cohort $(n=690)$. (B) Flow chart depicting SLN prediction algorithm and outcomes for T1 or T2 patients $(n=403)$ within a retrospective patient cohort $(n=690)$. In both panels, patients with class 1 A results would avoid SLNB, whereas class $1 B, 2 A$ or $2 B$ would have the surgical procedure. Five-year MSS, OS, DMFS and RFS rates with $95 \%$ Cls are shown for each group. SLN status for class $1 \mathrm{~B}-2 \mathrm{~B}$ patients reflects pathological node status wherein SLNB was performed, whereas outcomes for class 1A patients are reported regardless of SLNB. DMFS: Distant metastasis-free survival; GEP: Gene expression profile; MSS: Melanoma-specific survival; OS: Overall survival; RFS: Recurrence-free survival; SLN: Sentinel lymph node; SLNB: Sentinel lymph node biopsy.

T1-T2 tumor had an even lower rate of SLN positivity (1.6\%; i.e., well within the NCCN "Do Not Recommend" category). Of note, patients 55-64 years of age with class 1A, T1-T2 tumors had a SLN positivity rate of 4.9\%, which approaches but is below the rate for recommending a discussion about the SLN procedure. This information could be also useful in discussions with patients in this age group.

Older patients account for a substantial proportion of CM incidence, and 60\% of melanoma-related deaths occur in patients $\geq 65$ years old. While older age is associated with a poorer prognosis, increasing age appears to be inversely associated with SLN positivity $[14,16,17,37,38]$, which indicates that the prognostic value of SLNB is limited 
in this population $[39,40]$. Our results show that patients with class $1 \mathrm{~A}, \mathrm{~T} 1-\mathrm{T} 2$ tumors who are $\geq 65$ years of age have an SLN positivity rate of $1.6 \%$, substantially lower than the threshold recommended by practice guidelines. In the retrospective cohort, patients with a T1-T2 tumor and a class 1A GEP result had excellent MSS, OS, DMFS and RFS rates, suggesting they could safely forgo a SLNB procedure. This information could be helpful in making decisions about the SLNB in a population with a higher frequency of comorbidities and for which the procedure already shows a lower yield.

Survival rates for class $1, \mathrm{~T} 1 / \mathrm{T} 2$ patients in the retrospective cohort support this approach, with MSS rates comparable to those observed in patients with T1a tumors for whom current guidelines do not recommend SLNB [2]. MSLT-I showed that observation versus planned SLNB does not affect MSS [4], while MSLT-II demonstrated that a delay in completion lymph node dissection does not adversely impact survival [7]. Based on these data, one would expect that clinical follow-up of class 1A patients, with low-intensity follow-up recommendations for the identification of secondary skin cancers and recurrences and lymphadenectomy for those few who develop clinically detectable nodal disease, should achieve similar outcomes to those who currently undergo a planned SLNB. However, it is important to stress that follow-up for this low-risk group should be done according to current management guidelines and taking into account the patient's probability of recurrence (per NCCN recommendations) [6]. Patients for whom the decision is made to forgo SLNB should still have follow-up that includes clinical examination of the lymph node basin to ensure that, although infrequent, nodal metastases are identified as early as possible.

\section{Limitations}

Limitations of this study include the fact that long-term follow up is not available for patients in the prospective cohorts, however outcomes were modeled in the retrospective cohorts which have long-term outcomes [32]. Another potential limitation is that the study did not include a significant number of T1b-T2 patients who might have been considered for SLNB, but who either decided not to undergo the procedure or the procedure was not performed due to medical contraindications. As with all innovative approaches, replication in additional patient cohorts is recommended, to confirm the validity of this approach. Thus, a second multi-center validation study is ongoing to evaluate patients with T1-T2 melanoma who were clinically tested with the 31-GEP test and their SLNB results. Additionally, prospective, multi-center studies to track and evaluate clinical outcomes of patients for whom the 31-GEP test is used to guide SLNB decisions under IRB-approved protocols are planned. Based on results of the MSLT-I trial it is expected that class 1A patients with T1-T2 tumors who forgo the SLNB procedure will not demonstrate a significant difference in melanoma-specific survival rates compared with those who are managed with SLNB [4].

\section{Conclusion}

Incorporation of molecular signatures to guide biopsy recommendations is now routine for patients with thyroid, prostate and lung cancers [41-43]. Our results show that a gene expression signature can be applied in melanoma to identify a patient population with $<5 \%$ predicted probability of a positive SLN with demonstrated high survival rates and therefore has potential utility in guiding SLNB decisions. Additional multi-center retrospective and prospective studies to confirm and expand these results are ongoing or planned. If used in this way, the 31-GEP test could potentially reduce a substantial proportion of SLNBs while still maintaining a robust survival rate in those patients with low-risk tumor biology. These patients could benefit from avoidance of risks associated with surgery and anesthesia. Sentinel lymph node guidance using the GEP test is not meant to deter patients from surgical consultation, as it is always important to discuss all risks/benefits in an individual clinical situation. Rather, this test may serve as an additional decision-making tool for adhering to national recommendations of personalized care.

\section{Future perspective}

Over the next 5 to 10 years we expect that prognostication in melanoma will continue to evolve and incorporate molecular features of the tumor with traditional clinicopathologic features in order to best estimate individual risk, a strategy that has proven successful in other cancers. This is critical to defining informed clinical care strategies that benefit patients. With further delineation of risk profiles and continued therapeutic advances in the adjuvant and metastatic settings, melanoma patient care paradigms will continue to shift, particularly regarding the utilization of surgical intervention such as with completion lymph node dissection. Determination of the risk of SLN positivity is an example for which additional objective information is warranted. Given the reported rates of SLN positivity 
that range from $5-12 \%$ in $\mathrm{T} 1(\leq 1 \mathrm{~mm})$ melanomas, and $15-20 \%$ in tumors of intermediate thickness, there will clearly be a clinical benefit to focus the SLNB surgical procedure in the group that is most likely to benefit from it. We expect that the incorporation of gene expression profiling information for better delineation of those who have lower and higher risks of SLN positivity will have a substantial impact on clinical decision-making in the years ahead. We expect that this paradigm shift will lead to improved patient care while facilitating efficient and appropriate use of healthcare resources.

\section{Summary points}

- National guidelines recommend a threshold of $5 \%$ positivity risk for considering sentinel lymph node (SLN) biopsy.

- Most patients have a negative SLN result, including older patients who have poorer prognoses despite lower SLN positivity.

- There is a clinical need to identify patients who are likely to have a negative SLN biopsy result in order to reduce surgical burden and cost.

- A validated 31-gene expression profile (31-GEP) test for prognostication estimates metastatic risk as low (class 1A) or high (class 2B).

- This study evaluated whether the 31-GEP test could identify patients who have a low risk of SLN positivity and favorable outcomes, and thus could safely avoid SLN biopsy.

- In a validation cohort of 1421 prospectively-tested patients, patients 55-64 and $\geq 65$ years with T1-T2 melanoma and class $1 \mathrm{~A}$ results had SLN positivity rates of 4.9 and $1.6 \%$.

- In a retrospective cohort of 690 patients, patients $\geq 55$ years with T1-T2 melanoma and class $1 \mathrm{~A}$ results had favorable recurrence-free, distant metastasis-free, overall and melanoma-specific survival rates.

- The 31-GEP test can identify patients with T1-T2 melanoma who have both a low risk of SLN positivity and a good prognosis, suggesting this test can help address the clinical need of identifying patients at low risk for a positive SLN and can inform SLNB decisions.

\section{Supplementary data}

To view the supplementary data that accompany this paper please visit the journal website at: https://www.futuremedicine.com/d oi/suppl/10.2217/fon-2018-0912

\section{Acknowledgements}

The authors would like to acknowledge SJ Kurley, KM Plasseraud, CE Johnson and TM Poteet (Castle Biosciences, Inc.) for contributing to this study.

\section{Author contributions}

FA Monzon and RW Cook conceived and designed the study. All authors contributed to acquisition of the data. JT Vetto, FA Monzon and RW Cook performed analysis and interpretation of the data, and drafted the manuscript. All authors critically revised and approved the manuscript.

Financial \& competing interest disclosure

Castle Biosciences, Inc. funded this study. Castle Biosciences, Inc. drafted the study design and oversaw the data collection, management and all analyses. Castle Biosciences, Inc. contributed to data interpretation; preparation, review and approval of the manuscript. P Gerami is a paid consultant for Castle Biosciences, Inc. MD Fleming received an honorarium from Castle Biosciences, Inc. JT Vetto, EC Hsueh, BR Gastman, JD Wayne, AC Berger are on the speakers bureau for Castle Biosciences, Inc. JT Vetto, P Gerami and EC Hsueh received travel/meeting funding unrelated to this study from Castle Biosciences, Inc. JT Vetto, EC Hsueh, BR Gastman, LD Dillon, J Keller, X Huang, A Fleming, P Hewgley, P Gerami, S Leachman, JD Wayne, AC Berger and MD Fleming received funding for sample and/or clinical data acquisition and processing for this study. P Gerami and JT Vetto received travel funding to present data related to this study at a meeting and/or other purposes. FA Monzon and RW Cook are employees and options holders at Castle Biosciences, Inc. The authors have no other relevant affiliations or financial involvement with any organization or entity with a financial interest in or financial conflict with the subject matter or materials discussed in the manuscript apart from those disclosed.

No writing assistance was utilized in the production of this manuscript. 


\section{Ethical conduct of research}

The authors state that they have obtained appropriate institutional review board approval for all human studies. In addition, for investigations involving human subjects, informed consent has been obtained from the participants involved where required.

\section{Open access}

This work is licensed under the Attribution-NonCommercial-NoDerivatives 4.0 Unported License. To view a copy of this license, visit http://creativecommons.org/licenses/by-nc-nd/4.0/

\section{References}

Papers of special note have been highlighted as: $\bullet$ of interest; $\bullet \bullet$ of considerable interest

1. Siegel RL, Miller KD, Jemal A. Cancer statistics, 2018. CA Cancer J. Clin. 68(1), 7-30 (2018).

2. Gershenwald JE, Scolyer RA, Hess KR et al. Melanoma staging: evidence-based changes in the American Joint Committee on Cancer eighth edition cancer staging manual. CA Cancer J. Clin. 67(6), 472-492 (2017).

3. SEER. National Cancer Institute (2017).

4. Morton DL, Thompson JF, Cochran AJ et al. Final trial report of sentinel-node biopsy versus nodal observation in melanoma. N. Engl. J. Med. 370(7), 599-609 (2014).

-. Final results of the Multicenter Selective Lymphadenectomy I (MSLT-I) trial in which patients were randomized to observation or sentinel lymph node biopsy (SLNB). Study results demonstrate SLNB provides prognostic information but the procedure does not improve melanoma-specific survival.

5. Wong SL, Faries MB, Kennedy EB et al. Sentinel lymph node biopsy and management of regional lymph nodes in melanoma: American Society of Clinical Oncology and Society of Surgical Oncology Clinical Practice Guideline update. J. Clin. Oncol. 36(4), 399-413 (2018).

6. Coit DG, Thompson JA, Albertini MR et al. Melanoma, Version 3.2018, NCCN Clinical Practice Guidelines in Oncology. (2018).

-• Expert recommendations for SLNB suggesting the procedure should not be performed in patients who have $<5 \%$ risk of SLN positivity. These recommendations suggest that SLNB can be discussed and considered for patients with T1a melanoma and high risk features, such as mitotic rate $\geq \mathbf{2} / \mathbf{m m}^{2}$, lymphovascular invasion, or uncertain microstaging, and those with T1b melanoma.

7. Faries MB, Thompson JF, Cochran AJ et al. Completion dissection or observation for sentinel-node metastasis in melanoma. N. Engl. J. Med. 376(23), 2211-2222 (2017).

-• Results of the Multicenter Selective Lymphadenectomy Trial II (MSLT-II) in which patients were randomized to observation or completion lymph node dissection after a positive SLNB. There was no melanoma-specific survival benefit associated with the CLND procedure.

8. Eggermont AM, Chiarion-Sileni V, Grob JJ et al. Adjuvant ipilimumab versus placebo after complete resection of high-risk stage III melanoma (EORTC 18071): a randomised, double-blind, Phase 3 trial. Lancet Oncol. 16(5), 522-530 (2015).

9. Eggermont AM, Chiarion-Sileni V, Grob JJ et al. Prolonged survival in stage III melanoma with ipilimumab adjuvant therapy. N. Engl. J. Med. 375(19), 1845-1855 (2016).

10. Weber J, Mandala M, Del Vecchio M et al. Adjuvant nivolumab versus ipilimumab in resected stage III or IV melanoma. N. Engl. J. Med. 377(19), 1823-1835 (2017).

11. Long GV, Hauschild A, Santinami M et al. Adjuvant dabrafenib plus trametinib in stage III BRAF-mutated melanoma. N. Engl. J. Med. 377(19), 1813-1823 (2017).

12. Chang JM, Kosiorek HE, Dueck AC et al. Stratifying SLN incidence in intermediate thickness melanoma patients. Am. J. Surg. 215(4), 699-706 (2018).

13. Kachare SD, Brinkley J, Wong JH, Vohra NA, Zervos EE, Fitzgerald TL. The influence of sentinel lymph node biopsy on survival for intermediate-thickness melanoma. Ann. Surg. Oncol. 21(11), 3377-3385 (2014).

14. Cavanaugh-Hussey MW, Mu EW, Kang S, Balch CM, Wang T. Older age is associated with a higher incidence of melanoma death but a lower incidence of sentinel lymph node metastasis in the SEER databases (2003-2011). Ann. Surg. Oncol. 22(7), 2120-2126 (2015).

15. Han D, Zager JS, Shyr Y et al. Clinicopathologic predictors of sentinel lymph node metastasis in thin melanoma. J. Clin. Oncol. 31(35), 4387-4393 (2013).

16. Sinnamon AJ, Neuwirth MG, Yalamanchi $\mathrm{P}$ et al. Association between patient age and lymph node positivity in thin melanoma. JAMA Dermatol. 153(9), 866-873 (2017).

- Study showing that SLNB positivity decreases with age and there is an increase in false negative rate in patients $\geq 65$ years of age.

17. Sondak VK, Taylor JM, Sabel MS et al. Mitotic rate and younger age are predictors of sentinel lymph node positivity: lessons learned from the generation of a probabilistic model. Ann. Surg. Oncol. 11(3), 247-258 (2004).

18. Mcmasters KM, Noyes RD, Reintgen DS et al. Lessons learned from the Sunbelt Melanoma Trial. J. Surg. Oncol. 86(4), 212-223 (2004).

19. Morton DL, Cochran AJ, Thompson JF et al. Sentinel node biopsy for early-stage melanoma: accuracy and morbidity in MSLT-I, an international multicenter trial. Ann. Surg. 242(3), 302-311; discussion 311-303 (2005). 
20. Moody JA, Ali RF, Carbone AC, Singh S, Hardwicke JT. Complications of sentinel lymph node biopsy for melanoma - a systematic review of the literature. Eur. J. Surg. Oncol. 43(2), 270-277 (2017).

21. Ascha M, Ascha MS, Gastman B. Identification of risk factors in lymphatic surgeries for melanoma: a national surgical quality improvement program review. Ann. Plast. Surg. 79(5), 509-515 (2017).

22. Bagaria SP, Faries MB, Morton DL. Sentinel node biopsy in melanoma: technical considerations of the procedure as performed at the John Wayne Cancer Institute. J. Surg. Oncol. 101(8), 669-676 (2010).

23. Agnese DM, Abdessalam SF, Burak WE Jr, Magro CM, Pozderac RV, Walker MJ. Cost-effectiveness of sentinel lymph node biopsy in thin melanomas. Surgery 134(4), 542-547; discussion 547-548 (2003).

24. Gerami P, Cook RW, Russell MC et al. Gene expression profiling for molecular staging of cutaneous melanoma in patients undergoing sentinel lymph node biopsy. J. Am. Acad. Dermatol. 72(5), 780-785, e783 (2015).

25. Gerami P, Cook RW, Wilkinson J et al. Development of a prognostic genetic signature to predict the metastatic risk associated with cutaneous melanoma. Clin. Cancer Res. 21(1), 175-183 (2015).

-. Study describing the initial development and validation of the 31-gene expression profile (31-GEP) test to predict metastasis in cutaneous melanoma.

26. Hsueh EC, Debloom JR, Lee J et al. Interim analysis of survival in a prospective, multi-center registry cohort of cutaneous melanoma tested with a prognostic 31-gene expression profile test. J. Hematol. Oncol. 10(1), 152 (2017).

-• First study showing results of the 31-GEP test in a prospective cohort that confirms its independent prognostic value.

27. Zager JS, Gastman BR, Leachman S et al. Performance of a prognostic 31-gene expression profile in an independent cohort of 523 cutaneous melanoma patients. BMC Cancer 18(1), 130 (2018).

28. Greenhaw BN, Zitelli JA, Brodland DG. Estimation of prognosis in invasive cutaneous melanoma: an independent study of the accuracy of a gene expression profile test. Dermatol. Surg. 44(12), 1494-1500 (2018).

29. Huang X, Hewgley WP, Guerrero W, Fleming MD. Application of gene expression profiling in the management of cutaneous melanoma. Society of Surgical Oncology Annual Meeting. Seattle, WA, Abstract PF308 (2017).

30. Hsueh EC, Schwartz TL, Lizalek JM, Hunborg PS, Hurley MY, Hinyard LJ. Prospective validation of gene expression profiling in primary cutaneous melanoma. J. Clin. Oncol. 34(15 Suppl.), 9565 (2016).

31. Dillon LD, Gadzia JE, Davidson RS et al. Prospective, multicenter clinical impact evaluation of a 31-gene expression profile test for management of melanoma patients. SKIN J. Cutan. Med. 2(3), 111-121 (2018).

32. Gastman BR, Gerami P, Kurley SJ, Cook RW, Leachman S, Vetto JT. Identification of patients at risk of metastasis using a prognostic 31-gene expression profile in subpopulations of melanoma patients with favorable outcomes by standard criteria. J. Am. Acad. Dermatol. 80(1), 149-157, e144 (2019).

-. Study describing the performance of the 31-GEP test in 690 patients with stage I-III melanoma. The outcomes in the current study are derived from this retrospective cohort.

33. Bilimoria KY, Balch CM, Wayne JD et al. Health care system and socioeconomic factors associated with variance in use of sentinel lymph node biopsy for melanoma in the United States. J. Clin. Oncol. 27(11), 1857-1863 (2009).

34. Hayek SA, Munoz A, Dove JT et al. Hospital-based study of compliance with NCCN guidelines and predictive factors of sentinel lymph node biopsy in the setting of thin melanoma using the national cancer database. Am. Surg. 84(5), 672-679 (2018).

35. Chen J, Xu Y, Zhou Y, Wang Y, Zhu H, Shi Y. Prognostic role of sentinel lymph node biopsy for patients with cutaneous melanoma: a retrospective study of Surveillance, Epidemiology, and End-Result population-based data. Oncotarget 7(29), 45671-45677 (2016).

36. Stiegel E, Xiong D, Ya J et al. Prognostic value of sentinel lymph node biopsy according to Breslow thickness for cutaneous melanoma. J. Am. Acad. Dermatol. 78(5), 942-948 (2018).

37. Balch CM, Soong SJ, Gershenwald JE et al. Age as a prognostic factor in patients with localized melanoma and regional metastases. Ann. Surg. Oncol. 20(12), 3961-3968 (2013).

38. Balch CM, Thompson JF, Gershenwald JE et al. Age as a predictor of sentinel node metastasis among patients with localized melanoma: an inverse correlation of melanoma mortality and incidence of sentinel node metastasis among young and old patients. Ann. Surg. Oncol. 21(4), 1075-1081 (2014).

39. Fleming NH, Tian J, Vega-Saenz De Miera E et al. Impact of age on the management of primary melanoma patients. Oncology 85(3), 173-181 (2013).

40. MacDonald JB, Dueck AC, Gray RJ et al. Malignant melanoma in the elderly: different regional disease and poorer prognosis. J. Cancer 2, 538-543 (2011).

41. Duick DS, Klopper JP, Diggans JC et al. The impact of benign gene expression classifier test results on the endocrinologist-patient decision to operate on patients with thyroid nodules with indeterminate fine-needle aspiration cytopathology. Thyroid 22(10), 996-1001 (2012).

42. Ferguson JS, Van Wert R, Choi Y et al. Impact of a bronchial genomic classifier on clinical decision making in patients undergoing diagnostic evaluation for lung cancer. BMC Pulm. Med. 16(1), 66 (2016).

43. Wojno KJ, Costa FJ, Cornell RJ et al. Reduced rate of repeated prostate biopsies observed in ConfirmMDx clinical utility field study. Am. Health Drug Benefits 7(3), 129-134 (2014). 